\title{
IN-VEHICLE NOISE ALTERS THE PERCEIVED MEANING OF AUDITORY SIGNALS
}

\author{
Neil Lerner ${ }^{1}$, Jeremiah Singer ${ }^{1}$, Daniel Kellman ${ }^{1}$, \& Eric Traube ${ }^{2}$ \\ ${ }^{1}$ Westat, Rockville, Maryland, USA \\ ${ }^{2}$ National Highway Traffic Safety Administration, Washington, DC, USA \\ Email: neillerner@westat.com
}

\begin{abstract}
Summary: Research on driver perception and interpretation of auditory signals has generally been conducted under conditions of low-to-moderate ambient in-cab noise. In a series of four experiments, the effects of various ambient noise conditions on the perceived meaning of auditory signals were investigated. Noise conditions that may be realistically anticipated in the course of normal driving altered the perceived urgency and meaning of signals. The presence and extent of such changes was a function of the specific auditory signal, the ambient noise condition, and their interaction. The results indicate that in-vehicle auditory signal design criteria developed only under low-to-moderate ambient noise conditions are not likely to be sufficient. The significant signal-by-ambient noise interaction further suggests that multiple noise backgrounds must be considered.
\end{abstract}

\section{OBJECTIVES}

Auditory signals are a primary means of warning drivers about high-urgency potential crash situations to which the driver must react quickly. Auditory signals may also be used to inform the driver about a variety of other situations, such as less urgent safety considerations, traffic information, vehicle status, or notification of incoming communications. As auditory signals become more prevalent during driving, it is essential that critical crash warnings be clearly discriminable from other sorts of messages. For an in-vehicle system of warnings, alerts, and notifications to be effective, signals for each type of message should convey their appropriate general meaning without specific driver training or experience. There are no standard sounds for in-vehicle warnings and different manufacturers use different signals for similar messages. There is opportunity for confusion when a signal is unfamiliar or is used in a manner different from the listener's past experience. Researchers have been addressing the features of auditory signals that lead to appropriate categorical perception (i.e, the sound naturally conveys the appropriate general category of meaning to a naive listener; Lewis, Eisert, Roberts, \& Baldwin, 2014). Such research may provide features or parameter boundaries that result in a given signal conveying the intended general message. In this way, crash warning signals convey high urgency and a need to react immediately while lower urgency messages will not result in inappropriate reactions.

Unfortunately, although considerable research has been undertaken on the design of auditory crash warnings and other messages for in-vehicle use, almost all of this research has been done under relatively benign ambient noise conditions. In actual practice, substantially louder ambient noise conditions may be expected to occur. Higher speeds and rougher road surfaces raise the noise level. There may be in-vehicle noise sources, such as music or children. Nearby heavy vehicle traffic may be loud. Windows may be lowered, with resulting loud buffeting noise. Heavy rain and wet road surfaces may result in substantial increases in sound level. Auditory messaging systems need to work well under conditions such as these as well as in more routine noise environments. In fact, some of the noisier ambient conditions may represent difficult 
driving conditions (e.g., heavy traffic, construction zones, inclement weather) where crash warnings may be particularly important. Field measurements made in recent model passenger vehicles by Lerner et al. (in publication) found ambient noise levels were in the mid-60s Aweighted decibel (dBA) range at $97 \mathrm{~km} / \mathrm{h}(60 \mathrm{mph})$ on a smooth asphalt highway, but increased by about $10 \mathrm{dBA}$ for various other conditions, and even more during moderately heavy rain. Yet many actual alerting signals in vehicles are at about 65 dBA (Lin \& Green, 2013). Although human factors guidelines typically call for higher levels of acoustic warnings, in actual practice they are often relatively low, presumably due to a desire to limit consumer annoyance. The ability of an auditory signal to maintain its effectiveness in ambient noise is a function of both its loudness and the particular properties of the sound. However, very little information exists on the perception of auditory vehicle warnings under noise conditions, including what such noise might do to the listener's interpretation of the meaning of the signals.

This paper describes some key findings from a series of experiments conducted as part of the National Highway Safety Traffic Administration's (NHTSA) program on Crash Warning Interface Metrics (CWIM). It examines driver perception of the meaning of auditory signals across a range of different auditory signals and various ambient noise conditions. Greater detail on the procedures and results may be found in forthcoming NHTSA CWIM reports on this work (Lerner et al., in publication; Singer, Lerner, Kellman \& Robinson, in publication).

\section{METHOD}

Four experiments were conducted using similar procedures. Each used different participants. The first was an on-road experiment in which participants periodically were presented with auditory signals while they were driving under various ambient noise conditions. The participants indicated when they heard the signal and then provided a series of ratings related to their perceptions of the signal. This on-road method was subsequently adapted to a laboratory method using high-quality binaural field recordings of in-vehicle noise and signals. The first laboratory experiment closely replicated the findings of the on-road experiment, thus validating the laboratory method. The final two laboratory experiments examined a broader range of ambient noise conditions, some additional auditory signals, signal intensity, and annoyance/acceptance of sounds for in-vehicle use. All recordings were made on a smooth asphalt roadway at $97 \mathrm{~km} / \mathrm{h}(60$ $\mathrm{mph}$ ) without adjacent traffic, except where indicated differently.

\section{On-Road Method}

Thirty-four participants (aged 22-49) engaged in normal driving on a limited access highway. Three ambient noise conditions were in effect during the drive: baseline, music (at participant's preferred listening level), and front windows down (i.e., both driver and front passenger windows fully lowered). During each ambient noise block, 15 auditory signals occurred in a random sequence (inter-trial intervals from 10-50 s, mean of $30 \mathrm{~s}$ ). When the participant detected a signal, they pressed a finger-worn microswitch, providing a detection response time. They then rated the signal on noticeability and on urgency (7 point scales) and then selected a category of meaning ("urgent crash warning," "safety information," "information not related to safety," or "incoming personal communication"). The 15 signals included 11 unique sounds at $65 \mathrm{dBA}$ plus four of these sounds also presented at $75 \mathrm{dBA}$. The sounds were adapted from current in-vehicle 
warnings and alerts, sounds from various sources, and synthetic speech. They provided a diverse set in terms of acoustic properties and intended meanings. Detailed descriptions of the sounds may be found in Lerner et al. (in publication). Three different vehicles were used as a betweengroups factor (a small car, sedan, and SUV). Since there was no significant main effect or interaction involving the vehicle type, this factor is not discussed further.

\section{Laboratory Method}

The laboratory method was a close analog of the on-road method. Lab methods offer advantages including greater experimental control, efficient data collection, and the ability to present transient noise conditions (e.g., rain, adjacent large vehicles). Actual driving was replaced by a simplified driving-like task in which participants maintained lateral and longitudinal control (using steering wheel and accelerator pedal) in a car-following task over a schematic road display on a computer screen. This required a sustained moderate level of attention to basic vehicle control, similar to that during the on-road drive. Other procedural aspects remained similar to that of the on-road experiment, except that the "music" ambient noise condition was fixed at $75 \mathrm{dBA}$. The number of ambient noise conditions and auditory signals varied across the three laboratory experiments (see below). Ambient noise recordings were made in a 2011 Toyota Camry using a Bruel and Kjaer Type 2270 sound level meter with Type 4101 binaural microphones worn by a researcher seated in the driver's seat. Playback in the laboratory was over Beyerdynamic DT-880 Pro (250 ohm) headphones. Details on recording, playback, and calibration methods may be found in Singer et al. (in publication). The same dependent measures were collected in the laboratory as on-road. In addition, the third laboratory experiment collected ratings of signal annoyance after each trial and ratings of the acceptability of the sound as an invehicle alert in a separate phase after the primary data collection.

Laboratory Experiment 1: Replication of on-road experiment. This experiment provided a direct replication of the on-road experiment, with 24 participants. It used the same 15 auditory signals and the same three ambient noise conditions (baseline, music, front windows down).

Laboratory Experiment 2: Comparison of ambient noise conditions. This experiment, with 36 participants, included a broader range of ambient noise conditions, including transient conditions. The seven noise conditions included the baseline and front windows down conditions of the previous experiment plus: concrete road surface; driver window half down; between trucks (semi-trailer trucks on both sides of subject vehicle); bumpy asphalt; heavy rain. Fifteen auditory signals were used in this experiment, all presented at $70 \mathrm{dBA}$. Ten of these were from the same set of the previous experiment and five were new examples.

Laboratory Experiment 3: Signal loudness. This experiment, with 24 participants, included six signals from the previous experiment, each presented at 65,70 , or $75 \mathrm{dBA}$. There were three different noise conditions: baseline, driver window half down, and heavy rain.

\section{SELECTED KEY FINDINGS}

In all four experiments, there were statistically significant main effects of the acoustic signal and the ambient noise condition, as well as a significant interaction of these two factors. Space 
limitations preclude presenting detailed data and inferential statistics for all of the dependent measures across four experiments; see Lerner et al. (in publication) and Singer et al. (in publication) for these details. What we present here are key findings that emerged across experiments and define the issues of concern for assuring that auditory communications systems in vehicles remain effective under the range of realistically-anticipated ambient noise conditions.

\section{On-the-Road Findings}

Figure 1 shows on-the-road findings for rated sound urgency under three noise conditions for each of 15 auditory signals. The first 11 sounds listed on the $\mathrm{x}$-axis were matched to be equally loud (equivalent to $65 \mathrm{dBA}$ pink noise) and the last four sounds were matched at $75 \mathrm{dBA}$. This figure illustrates a number of points: signals of subjectively similar loudness vary considerably in perceived urgency; music and especially windows-down noise conditions generally reduce perceived urgency; this effect is more substantial for some sounds than others; $75 \mathrm{dbA}$ sounds are generally perceived as more urgent than $65 \mathrm{dBA}$ sounds and are more resistant to ambient noise effects. However, some $65 \mathrm{dBA}$ sounds are seen as urgent and are relatively more resistant to noise effects. Similar findings were seen with respect to response time, rated noticeability, and category of meaning. This experiment established, under actual on-road driving conditions, that the interpretation of auditory signals is influenced by the noise backgrounds in which they occur. Figure 2 shows an example of how the signal meaning category may shift under different noise conditions. This example is for a blind spot warning at $75 \mathrm{dBA}$ that was generally seen as safetyrelated. Effects varied among signals. For some low-urgency signals, there was an increase in the tendency to see the signal as safety-related as discriminability was reduced in noise.

\section{Validity of the Laboratory Method}

Laboratory Experiment 1 very closely replicated the on-road findings, thus validating the laboratory method. Table 1 shows that the correlations for noticeability, urgency, and meaning under each noise condition were quite high, ranging from $r=0.84$ to 0.96 . Correlation coefficients for percent detected, while strong, were somewhat lower, particularly for "windows down." This was likely due to the greater uniformity in ambient noise conditions in the laboratory.

\section{Comparison of Ambient Noises}

Some noise conditions resulted in substantial changes in perception relative to the baseline condition. Figure 3 (from Lab Experiment 2) shows the group mean ratings for each noise condition, averaged across 15 auditory signals. Since some signals were more impervious to noise than others, some signals showed greater ambient noise effects than the mean effects shown in the figure. All signals were at $70 \mathrm{dBA}$, so that greater effects would be expected as compared to $65 \mathrm{dBA}$. In particular, heavy rain and windows down had substantial effects.

\section{Interaction of Auditory Signal and Ambient Noise}

Figure 4 (from Lab Experiment 2) shows one example of the interaction effect of auditory signal with ambient noise. The "pedestrian warning" signal lost urgency under the front windows down 
condition, but not under heavy rain. The "male voice" signal lost a great deal of urgency under front windows down, but only about one-third as much under heavy rain. In contrast, a signal (labeled "GMU 1") devised by researchers at George Mason University to meet certain key criteria for crash warning alarms, was essentially unaffected by front windows down but lost urgency under rain. This indicates that the resistance of an auditory signal to noise effects is not an attribute of the signal alone, but rather includes its interaction with the specific characteristics of the background noise.

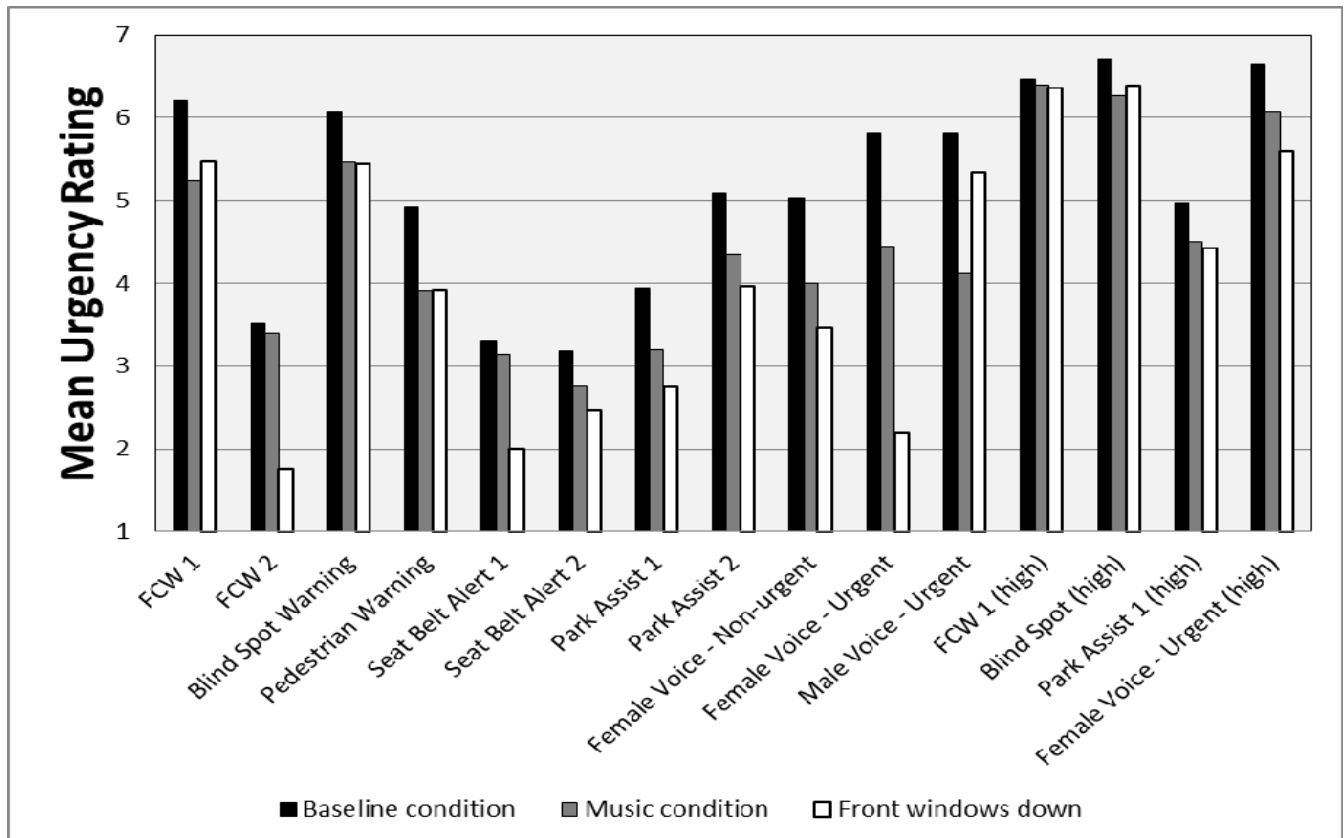

Figure 1. Mean on-road urgency ratings for each combination of signal and ambient noise condition

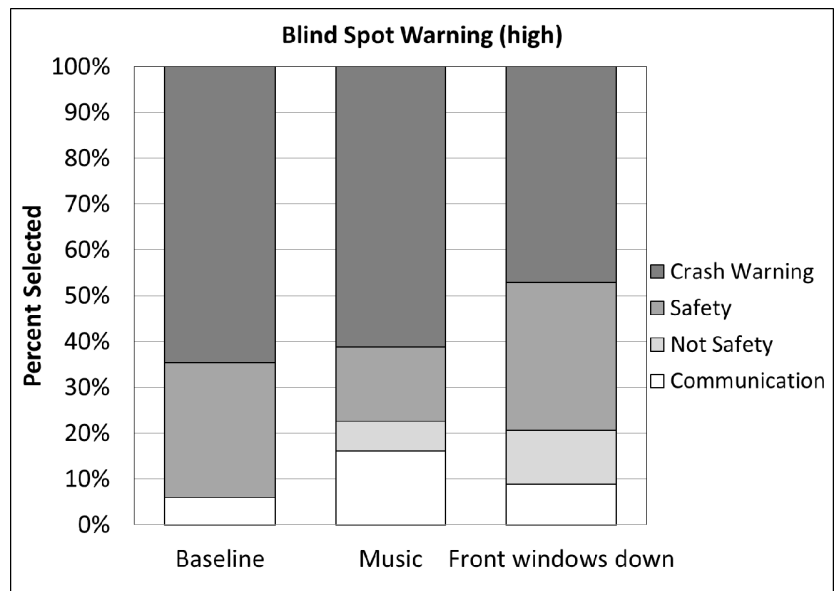

Figure 2. Shifts in signal meaning categorization under noise conditions

\section{Signal Intensity}

As anticipated, auditory signal intensity influenced perception of the signal. Figure 5 (from Lab Experiment 3 ) shows rating data (averaged across six signals) as a function of sound pressure level under each of three ambient noise conditions. Noticeability, urgency, and annoyance all 
increase with increases in signal intensity. However, the functions become steeper as ambient noise increases. Ratings of signals at $65 \mathrm{dBA}$ are much lower than ratings at $75 \mathrm{dBA}$ in heavy rain but only slightly lower under baseline conditions. Thus the benefits of louder signals are not particularly evident until more disruptive ambient noise conditions are considered.

Table 1. Correlation coefficients between on-road and laboratory findings

\begin{tabular}{|l|c|c|c|}
\hline Dependent Measure & Baseline Noise & Music On & Front Windows Down \\
\hline Percent detected & Not applicable & 0.837 & 0.642 \\
\hline Noticeability & 0.950 & 0.944 & 0.925 \\
\hline Urgency & 0.951 & 0.937 & 0.942 \\
\hline Meaning & 0.956 & 0.884 & 0.866 \\
\hline
\end{tabular}

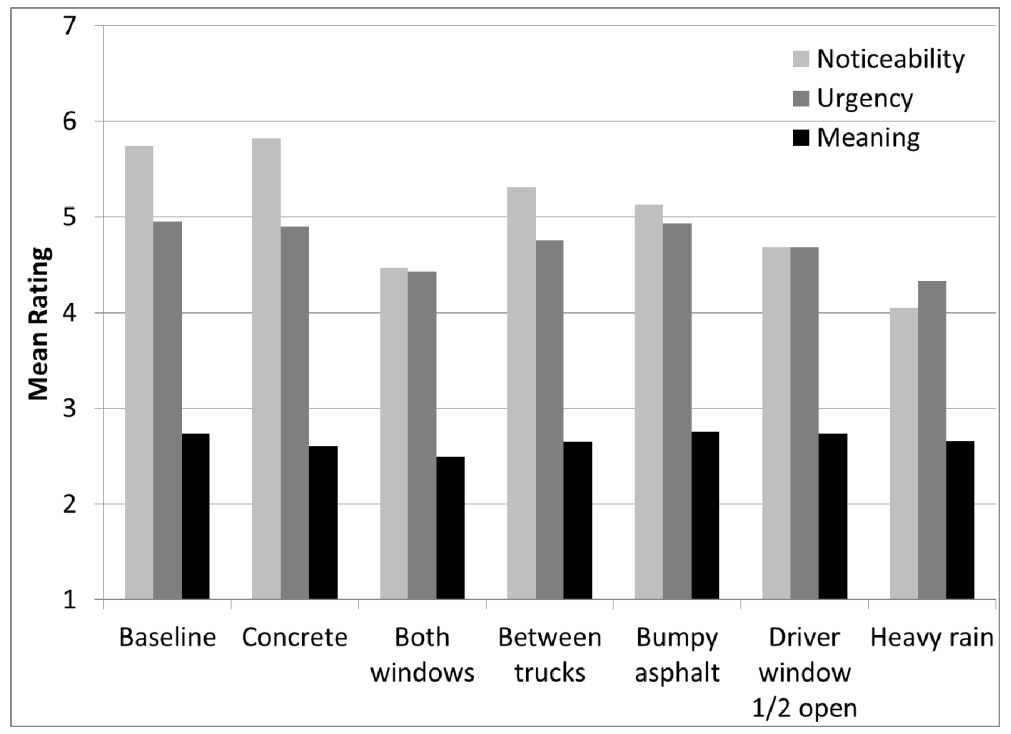

Figure 3. Mean ratings (across 15 signals) under various ambient noise conditions

\section{CONCLUSIONS}

This set of experiments demonstrated that foreseeable ambient noise conditions can alter driver perception of the meaning of auditory signals. Signals of similar loudness can vary substantially in their resistance to noise effects. Many signals presented at $65 \mathrm{dBA}$ (often seen in practice) may fail even to be heard in various noise environments. Higher urgency signals can lose urgency under noise, even if reliably heard. Signals perceived as non-urgent under low noise may become more ambiguous in higher noise. Thus a coordinated system of auditory messages may be degraded under foreseeable driving conditions. In developing design and evaluation criteria, it is insufficient to simply employ low noise conditions. Some ambient noise conditions appear particularly challenging (e.g., rain, front windows down). One key finding is that there is a meaningful interaction between the auditory signal and the particular noise condition. This means that in comparing and evaluating auditory signals, it is not sufficient to simply look at "low" and "high" noise backgrounds. One signal may be degraded more by one noise condition while another signal is degraded more by a different noise condition. Although this research observed substantial differences among auditory signals, it did not systematically manipulate acoustic parameters and features to develop specific recommended design criteria. Lewis et al. (2014) provide a model for such systematic consideration of critical signal parameters. 


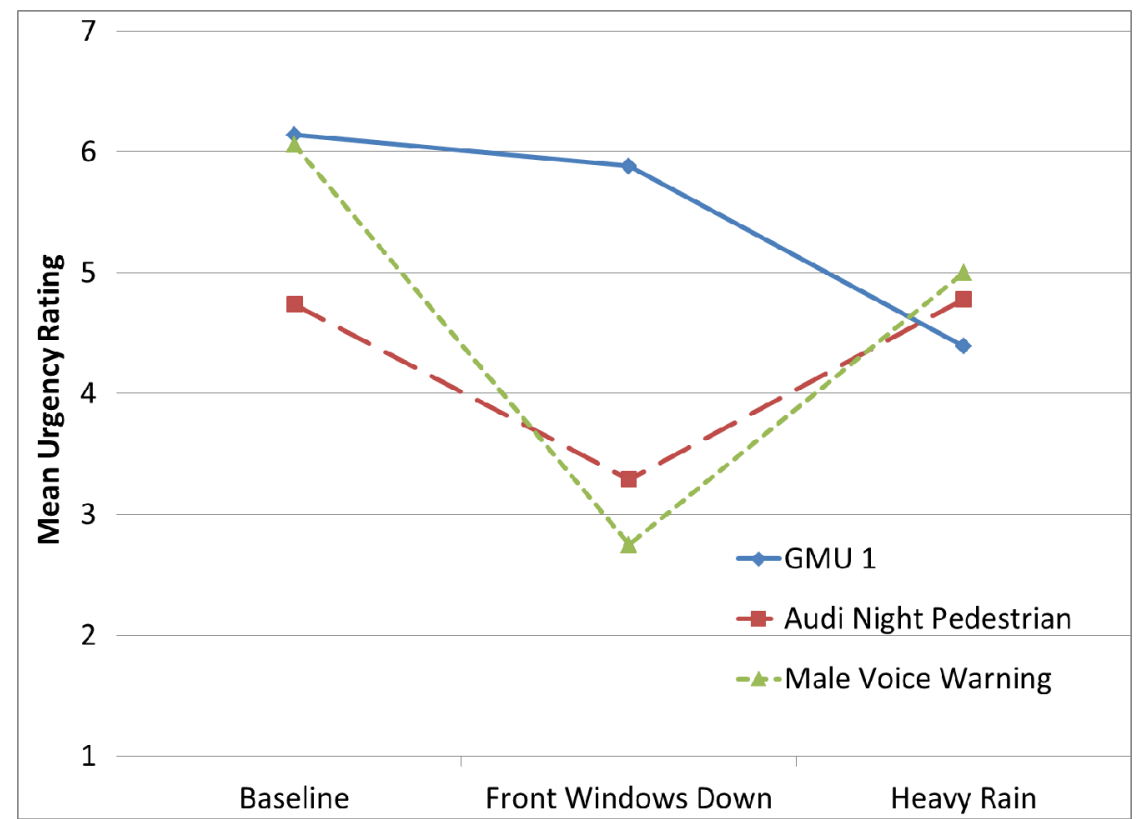

Figure 4. Differing effects of rain and windows down ambient noise conditions on perceived urgency of three different warning signals
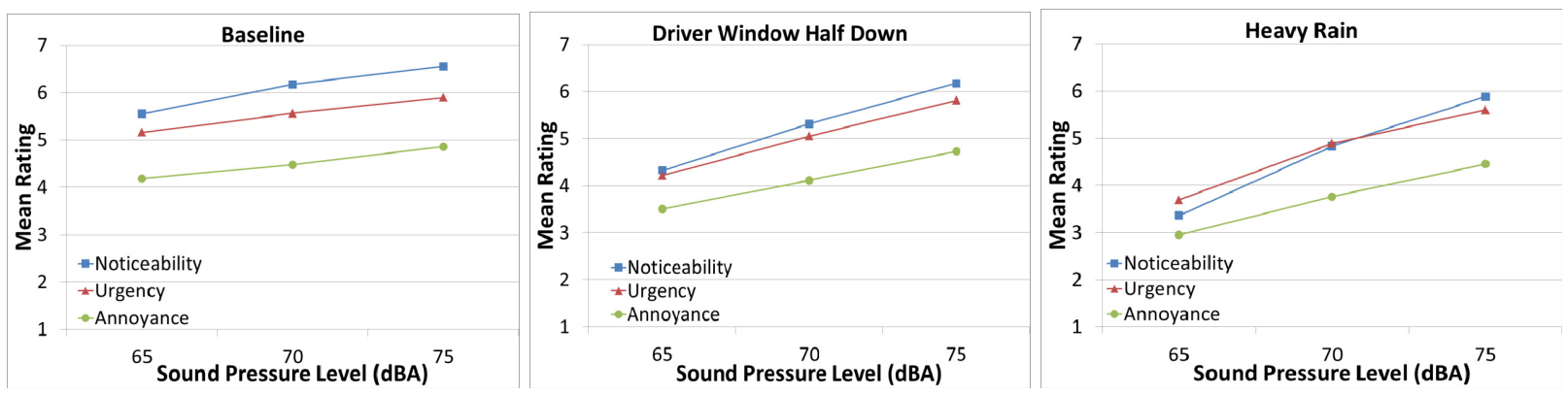

Figure 5. Main effects of loudness on mean ratings for each ambient noise condition

\section{REFERENCES}

Lerner, N., Singer, J., Huey, R., Brown, T., Marshall, D., Chrysler, S., Schmitt, R., Baldwin, C.L., Eisert, J.L., Lewis, B., Bakker, A.I., \& Chiang, D. (in publication). Crash Warning Interface Metrics (CWIM): Phase 3 Final Report. Washington, DC: National Highway Traffic Safety Administration.

Lewis, B., Eisert, J., Roberts, D., and Baldwin, C. (2014). Designing unambiguous auditory crash warning systems. Proceedings of the 2014 International Annual Meeting of the Human Factors and Ergonomics Society, Chicago, Illinois, 2078-2082.

Lin, B. and Green, P. (2013). Measurements of Driver-Assistance Warning-Signal Characteristics in 2013 Cars. Report No. UMTRI-2013-03. Ann Arbor, Michigan: University of Michigan Transportation Research Institute.

Singer, J., Lerner, N., Kellman, D., and Robinson, E. (in publication). Warning and Message Perception Under Ambient Noise Conditions: Laboratory Experiments. Report under Contract DTNH22-11-D-00237/002. Washington, DC: National Highway Traffic Safety Administration. 